\title{
Outil d'apprentissage à la gestion des mesures préventives en santé et sécurité du travail basé sur les coûts
}

Learning Tool for a Cost-Based Management of Occupational Health and Safety Measures

Sylvie Gravel, Jessica Dubé et Carole Sylvestre

\section{(2) OpenEdition Journals}

\section{Édition électronique}

URL : http://journals.openedition.org/pistes/6498

DOI : 10.4000/pistes. 6498

ISSN : 1481-9384

\section{Éditeur}

Les Amis de PISTES

\section{Référence électronique}

Sylvie Gravel, Jessica Dubé et Carole Sylvestre, «Outil d'apprentissage à la gestion des mesures préventives en santé et sécurité du travail basé sur les coûts », Perspectives interdisciplinaires sur le travail et la santé [En ligne], 22-1 | 2020, mis en ligne le 01 juin 2020, consulté le 21 avril 2021. URL: http://journals.openedition.org/pistes/6498; DOl : https://doi.org/10.4000/pistes.6498

Ce document a été généré automatiquement le 21 avril 2021.

\section{(c)}

Pistes est mis à disposition selon les termes de la licence Creative Commons Attribution - Pas d'Utilisation Commerciale - Pas de Modification 4.0 International. 


\section{Outil d'apprentissage à la gestion des mesures préventives en santé et sécurité du travail basé sur les coûts}

Learning Tool for a Cost-Based Management of Occupational Health and Safety

Measures

Sylvie Gravel, Jessica Dubé et Carole Sylvestre

La santé et la sécurité du travail (SST) est une problématique ayant des incidences financières pour les organisations, les employés et la société (Gosselin, 2004). L'analyse des coûts de la gestion de la SST peut être abordée selon différentes perspectives, celles des travailleurs, des gestionnaires, des assureurs privés et des institutions publiques qui contribuent au rétablissement des travailleurs souffrant de lésions professionnelles, comme le système de santé et les services d'indemnisation et de réadaptation (Tompa, Verbeek, Van Tulder et De Boer 2010; Boulenger et Vaillancourt, 2012). Depuis les premiers travaux de H.W. Heinrich en 1931, l'analyse des coûts de SST a évolué selon deux grands axes : a) l'approche macroéconomique centrée sur l'évolution des coûts selon la transformation des régimes comme l'inclusion de nouveaux programmes (ex. travailleuses enceintes) ou de nouvelles normes (ex. soins en physiothérapie); b) l'approche microéconomique centrée sur l'entreprise et ses acteurs (employeurs, employés et syndicat). En parallèle au développement de ces deux axes, la recherche s'est également centrée sur l'analyse coût-bénéfice et plus particulièrement sur les coûts assumés par les entreprises : a) les projets d'investissement à la prévention ; b) les coûts liés à un événement accidentel; c) les coûts de réparation; d) les coûts de performance organisationnelle (Gosselin, 2004; Fulmiler, 2000). Pour procéder à ces analyses, les auteurs ont élaboré des typologies des coûts directs, indirects et humains (Gosselin, 2004 ; Lebeau, Duguay et Boucher, 2014). De façon complémentaire, d'autres travaux ont développé des outils d'aide à la décision d'investir ou non dans les mesures et les programmes préventifs comme les programmes de prévention des troubles musculo-squelettiques, des chutes, etc. (Lee, 2017). Bien que les évaluations économiques devraient, en principe, contribuer au processus décisionnel, d'autres 
facteurs que l'efficacité économique dictent la décision finale, comme la rétention du personnel difficilement remplaçable (Hoch et Dewa, 2005 ; Redinger et Levine, 1998).

2 Les analyses macroéconomiques servent à estimer les coûts globaux des lésions professionnelles. En 2010, le gouvernement canadien estimait que les indemnisations réclamées pour des accidents du travail et maladies professionnelles étaient de 10 milliards de dollars en coûts directs et de 9 milliards de dollars en coûts indirects (Gilks et Logan, 2010). Au Québec, en 2015, les coûts directs et indirects étaient estimés à 4,62 milliards de dollars (Lebeau, 2015). Lebeau, Duguay et Boucher (2014) ont estimé, en se référant aux lésions indemnisées par la CNESST du 1er janvier 2005 au 31 décembre 2007, que le coût moyen d'une lésion professionnelle était de 38355 \$, incluant les coûts directs, indirects et humains. Le coût d'un accident du travail était estimé à 32848 \$ et grimpait à $161017 \$$ pour une maladie professionnelle et à 2,5 millions de dollars dans le cas d'un décès. Même si globalement la fréquence des lésions a baissé au fil des années, ces analyses démontrent la croissance des coûts liée à l'augmentation de la gravité des lésions (CNESST, 2016).

3 Selon les données les plus récentes au Québec de 2016 à 2018, les lésions indemnisées ont augmenté de $15 \%$, passant de 90414 à 103 406, exerçant une pression sur les coûts directs, faisant passer pour la même période les dépenses d'indemnisation de 2 à 2,2 milliards de dollars, excluant dans ce calcul tous les coûts indirects (Lanoie, Rivard, Fortin, Bernard et coll., 2019). Certes, il est hasardeux de comparer les résultats de ces études étant donné qu'elles sont basées sur des méthodes d'analyse et des typologies de coûts différentes. Toutefois, il s'agit d'analyses globales, dont il est possible d'extraire des estimations de coûts selon les diagnostics posés, les sites corporels atteints, les secteurs économiques et la nature de l'accident ou l'apparition des symptômes. Bien qu'intéressantes, ces données sur l'ensemble des coûts directs et indirects de SST interpellent peu les gestionnaires d'entreprise, dont l'intérêt premier est les coûts assumés par leur entreprise en matière d'absence, de perte de productivité, d'expertise et de perte de parts de marché.

4 L'analyse comparée des études microéconomiques est plus difficile, voire impossible, puisque les critères d'analyse des coûts-bénéfices sont rarement identiques comme pour les investissements de programmes ergonomiques ou de formation sur les méthodes sécuritaires (Lee, 2017; Tompa, Dolinschi, de Oliveira, 2006). De plus, rarement l'ensemble des coûts directs, indirects et humains est pris en compte dans les études. Certains auteurs retiennent certains coûts et d'autres pas, et les périodes d'observation sont souvent trop courtes pour voir les changements se produire (Cadieux, Roy et Desmarais, 2004 ; Champoux et Brun, 2010; Vinet, 2015). Comme le soulignent Tompa et coll., (2008), les promoteurs de programmes de prévention, convaincus des mérites financiers de leur programme, vont trop souvent retenir des critères d'appréciation qui avantagent l'évaluation de leur programme.

D'autres auteurs considèrent que le raisonnement coûts-bénéfices mise avec justesse sur les effets à long terme, mais déplorent que les gestionnaires d'entreprises soient au contraire centrés sur une gestion à court terme, sur les gains rapides des actions immédiates (Champoux et Brun, 2010; Frick et Kempa, 2011; Roy, Cadieux et Desmarais, 2005; Sultan-Taïeb, 2011 ; Vinet 2015). En fait, l'horizon temporel de l'évaluation coûts-bénéfices dépend de la perspective des acteurs réclamant cette analyse : l'entreprise, les travailleurs et leur famille ou la société en général (Tompa et coll., 2010). 
6 Aux fins de cet article, l'analyse microéconomique centrée sur les entreprises et les travailleurs a été privilégiée. Parmi les études recensées, les coûts les plus souvent rapportés par les entreprises sont les coûts d'accidents et les coûts de maladies récurrentes comme les troubles musculo-squelettiques. Initialement, les travaux de Grimaldi et Simonds (1989) ont apporté une contribution importante à la classification des coûts assumés par les entreprises avec leur typologie distinguant quatre types d'accidents: a) ceux sans perte de temps; b) ceux nécessitant l'intervention d'un médecin; c) ceux nécessitant des premiers soins; d) ceux avec perte de temps. Cette typologie permet de moduler les coûts selon la fréquence et la gravité des événements, dans l'analyse des coûts totaux des lésions professionnelles au sein d'une entreprise. Dans le même ordre d'idées, Laufer (1987) observe, bien des années plus tard, que si l'analyse des coûts selon la fréquence est incluse dans les coûts directs et celle de la gravité dans les coûts indirects, et que la somme de ces deux coûts est comparée aux coûts fixes des primes d'assurance, cette méthode de calcul est un incitatif important à la prise de décision d'investir en prévention.

7 L'analyse des coûts assumés par les entreprises s'est raffinée au fil des années pour, entre autres, tenir compte des coûts assujettis aux programmes de réadaptation (Jallon et coll., 2011). Au départ, les analyses de coûts se centraient sur la perte salariale, maintenant, elles incluent les pertes de salaires consécutives aux incapacités temporaires, permanentes et partielles, pouvant s'étaler sur quelques années (Boden et Galizzi, 1999). Par exemple, à la fin des années 90, LaBelle (2000) détermine les coûts des accidents du travail d'une entreprise en ajoutant les coûts indirects liés aux journées de travail réduites et perdues, les invalidités permanentes ainsi que les coups évités et les dommages matériels. Des analyses qui ont permis de démontrer des inégalités de genre et de conditions socioéconomiques chez les travailleurs souffrant de lésions. Des analyses qui ont mené à circonscrire les coûts humains, plus difficilement quantifiables, mais qui affectent quotidiennement la qualité de vie des travailleurs souffrant de lésion (Lanoie, 2002).

8 Toutefois, comme le démontre Dorman (2000), l'analyse des coûts des lésions professionnelles peut être faussée lorsque les coûts sont externalisés comme la soustraitance des emplois à risque ou la sous-déclaration des lésions, et les invalidités déguisées en chômage. Deux stratégies d'évitement des coûts de lésions professionnelles assumés par les employeurs qui seront transférés aux travailleurs et leur famille. Malgré le raffinement des analyses, il demeure difficile de recenser tous les coûts et de les mettre dans une équation efficiente pour les gestionnaires, surtout s'ils assument quotidiennement de multiples tâches comme c'est le cas dans les petites et moyennes entreprises. Les typologies de coûts ont évolué au cours des années, celle qui est le plus fréquemment reprise est celle comprenant trois types de coûts : directs, indirects et humains (Lebeau et Duguay, 2011). Toutefois, il n'existe pas de consensus, des auteurs classent certains coûts dans les coûts directs alors que d'autres auteurs classent les mêmes éléments dans les coûts indirects. Le présent projet a adopté une définition des coûts directs et indirects qui visait à faciliter la réalisation des objectifs de l'étude, écartant ainsi les coûts humains. 


\section{Problématique}

9 L'analyse macroéconomique et microéconomique des coûts des lésions professionnelles (coûts globaux) s'est perfectionnée, démontrant l'ampleur du problème. Toutefois, l'analyse microéconomique des coûts de SST à l'échelle des entreprises présente encore de nombreuses lacunes (Bräunig et Kohstall, 2011). Les études portant sur la gestion des coûts de SST dans les entreprises ont abordé le sujet sous différents angles: a) la disponibilité des données au sein des entreprises ; b) l'analyse a priori et posteriori des coûts, et c) les outils de comptabilisation des coûts.

Les études portant sur la disponibilité des données selon les départements et les niveaux hiérarchiques dans l'entreprise démontrent que les équipes de production identifient aisément les coûts directs entre autres liés aux absences et aux remplacements de personnel et d'équipements, alors que les coûts indirects liés aux pertes de productivité étaient plus aisément circonscrits par la haute direction (Jallon et coll., 2011).

11 Les analyses a priori et a posteriori mettent en perspective les économies relatives découlant des investissements préventifs (Gosselin, 2004 ; Sultan-Taïeb, 2011). Les coûts a priori se composent essentiellement des coûts de prévention et d'évaluation associés à l'implantation d'un nouvel équipement ou procédé de production, dont l'appréciation est basée sur les performances de production avant et après (Gosselin, 2004). Les coûts a posteriori sont plus larges que les économies liées à la prévention des accidents, ils englobent également les primes d'assurance (indemnité et bris d'équipement), les coûts d'enquête, les coûts de remplacement du personnel et autres dépenses découlant des séquelles physiques et morales subies par les travailleurs blessés et par le collectif de travail (Gosselin, 2004).

Deux principales approchesse dégagent des études portant sur les outils de comptabilisation des coûts par les entreprises : a) les logiciels de gestion de la qualité de la production incluant les coûts de SST; et b) le libre accès en ligne à des outils d'aide à la comptabilisation des coûts de SST. Certaines entreprises, surtout les grandes entreprises qui se sont dotées d'un système de gestion informatisé de la qualité, incluent dans le cycle de production des indicateurs de perte de temps, de perte de produits (brisés, défectueux, non conformes) et de perte de parts du marché (Berrhouma et coll., 2009). Selon ces derniers, les dépenses investies pour contrôler les coûts d'évaluation et de prévention de la qualité de nouveaux projets se situent entre $23 \%$ et $34 \%$ du coût global de développement. Une dépense a priori non négligeable pouvant freiner bien des investissements si ceux-ci sont perçus comme dédiés uniquement à la prévention de la SST. L'investissement doit aussi contribuer à l'amélioration de la productivité, sinon le statu quo est probablement évalué moins coûteux que d'investir dans la prévention.

D'autres études se sont intéressées à la comptabilisation démocratique des coûts de SST : l'accès à une application en ligne d'un outil convivial pour toutes les entreprises ne disposant de peu ou pas de système informatisé de gestion. C'est dans cette perspective qu'en 2010 l'« Institute for Work \& Health » de l'Ontario a mis en ligne le logiciel «Health \& Safety Smart Planner $»^{1}$. Il s'agit d'un calculateur disponible gratuitement en ligne, qui inclut dans ses estimations de coûts le temps perdu par le travailleur et son équipe, les coûts de la rémunération des travailleurs et des gestionnaires concernés, les dépenses de productivité et toutes les dépenses liées aux 
bris de matériaux (Tompa et coll., 2010). Testée auprès de deux groupes de gestionnaires, l'application est conviviale et facile pour quantifier les coûts et les avantages de la sécurité dans un plan d'affaires². Toutefois, le « Health \& Safety Smart Planner » est considéré trop complexe pour les gestionnaires de petites entreprises qui ne bénéficient pas du soutien d'un service comptable pour documenter ce type de coûts.

La gestion de la SST s'inscrit dans une logique décisionnelle dans la mesure où les gestionnaires sont habilités ou supportés pour recenser et calculer les coûts et pour comparer l'efficience des options d'investissements (Darbon et Letourmy, 1983; Gaudemet et Walliser, 1983 ; Brun et Lamarche, 2006). Néanmoins, les défenseurs d'une saine gestion de la SST oublient parfois que parmi les options possibles il y a celle de ne rien faire. Certaines entreprises sont tentées de miser sur le statu quo en espérant que la situation dangereuse ne se reproduira pas avant de nombreuses années (Laufer, 1987). Cette dernière option est souvent la plus économique, si l'entreprise n'est pas accusée de négligence.

Afin de soutenir l'argumentaire de développer des systèmes de gestion performant incluant la gestion de la SST, Redinger et Levine (1998) ont développé un modèle inspiré des systèmes ISO (International Organization for Standardization) pour l'assurance qualité (ISO 9001) et la gestion environnementale (ISO 14001), et de guides américains de prévention en santé et sécurité au travail comme celui de l'Occupational Safety and Health Administration (OSHA). Les experts consultés pour évaluer ce modèle constatent que le succès d'implantation d'un tel modèle repose sur la rétroaction systématique de tous événements ou incidents, rétroaction animée de façon continue par un gestionnaire. Selon Redinger et Levine (1998), ultimement, la question porteuse de changements est «pourquoi ?» : pourquoi les bris; pourquoi les lésions, pourquoi les absences ; pourquoi ces coûts? Selon ces auteurs, la rétroaction visant à répondre à ces questions est la pierre angulaire de la planification stratégique et du développement continu.

Pour Cadieux et Pérusse (2018), une entreprise qui désigne un gestionnaire dédié à la gestion de la SST manifeste de son engagement pour une culture forte de SST. L'entreprise peu concernée par les enjeux de la SST inscrit les coûts des accidents et des réparations comme des pertes inhérentes aux opérations. Les processus d'enquête tenteront davantage de trouver un coupable (une personne, un comportement ou une défectuosité du procédé), une démarche qui ne conduit à aucun apprentissage organisationnel, à aucune forme de résilience. Il y a aussi l'entreprise réactive qui investira dans la recherche d'une solution pour pallier les conséquences ou les menaces ayant engendré l'événement. Trop souvent, c'est l'accident grave qui obligera l'entreprise à s'extraire du statu quo en matière de prévention, en trouvant un équilibre entre obtenir des résultats et minimiser les pertes. Il y a aussi l'entreprise accommodante qui se contentera de répondre adéquatement aux exigences des lois et des normes, qui se satisfait d'une posture de conformité. Il y a aussi l'entreprise proactive en SST qui prend des initiatives, qui instaure un dialogue permanent avec ses employés et avec des ressources internes et externes pour bonifier sa gestion de la SST (SGSST), une démarche paritaire. Et finalement, il y a l'entreprise génératrice d'une pratique exemplaire, dont les valeurs de prévention doivent être partagées même avec les fournisseurs et les clients, un ensemble de valeurs qui s'inscrit dans toute la chaîne de production, quitte à modifier les procédés, les méthodes et les produits impliqués. 

rassembleur pour établir des assises pour une gestion paritaire de la SST, en mettant à contribution les syndicats (Ferron, 2013). L'expérience réussie de la gestion paritaire de la SST dans une entreprise québécoise a permis de faire des économies substantielles en réduisant les accidents de $70 \%$ sur une période de deux ans, et faisant passer le budget annuel de SST de 400000 \$à 65000 (Ferron, 2013).

Pour maximiser les gains scientifiques en matière de prévention de la SST, il faut soutenir les entreprises à devenir résilientes, savoir rebondir face aux menaces que peuvent représenter une lésion professionnelle et surtout lorsque ces lésions se répètent et s'aggravent (Gravel et coll. 2013). La résilience organisationnelle est le passage du statut quo, posture qu'adopte l'entreprise peu sensibilisée, à l'investissement proactif générateur de nouvelles pratiques préventives.

Pour ce faire, cette étude vise à élaborer et tester un outil d'apprentissage des compétences basé sur l'estimation des coûts de SST au sein de l'entreprise ; nous avons retenu trois éléments de succès de la gestion de la SST : a) la rétroaction fréquente portée de façon continue par un gestionnaire (Redinger et Levine, 1998); b) le développement d'une résilience organisationnelle lors de la survenue d'un événement accident ou d'une maladie grave (Gravel et coll., 2013, Cadieux et Pérusse, 2018) ; c) l'analyse coûts-bénéfices d'investissement en prévention et l'efficience de productivité de l'entreprise (Fulmiler, 2000).

Bien que les compétences des futurs gestionnaires (étudiants) pour estimer les coûts de SST sont multiples, comme l'illustre l'état des connaissances, nous avons centré nos efforts d'apprentissage sur quatre compétences: 1) identifier les éléments liés à l'événement accidentel ou la manifestation de la maladie; b) estimer les coûts d'événement et de réparation; c) anticiper les coûts pour des événements aggravés, alors que l'entreprise choisit de ne rien faire; d) développer un argumentaire adressé aux décideurs des entreprises (direction de département, conseil d'administration) pour investir en SST.

\section{Objectifs de recherche}

21 Afin de contribuer au développement de ces compétences de la gestion de la SST auprès des futurs gestionnaires (étudiants), le projet de recherche à l'origine de cet article visait à documenter les questions suivantes :

1. Quelles sont les composantes des coûts directs et indirects d'une lésion professionnelle que les gestionnaires en exercice peuvent identifier (compétence 1) et calculer facilement (compétence 2)?

- Parmi l'ensemble des coûts identifiés dans la littérature, lesquels sont facilement nommés par les gestionnaires en exercice lorsqu'ils relatent un événement accident ou un épisode de maladie?

1. Quelles sont les composantes des coûts directs et indirects que les futurs gestionnaires (étudiants) sont capables d'anticiper (compétence 3) liées aux conséquences d'une approche non préventive?

- Si l'entreprise décide de ne rien faire et qu'une autre situation plus grave se produit (compétence 4) ;

- Si l'entreprise décide de ne rien faire, entraînant une menace pour le collectif de travail, les clients, leurs parts de marché ou leur réputation (compétence 4).

Perspectives interdisciplinaires sur le travail et la santé, 22-1 | 2020 


\section{Cadre théorique et méthodologique}

Pour atteindre ce but et ces objectifs, nous avons retenu trois éléments de concepts : a) la résilience organisationnelle, centrée sur le passage d'une entreprise peu concernée à une entreprise proactive en matière de gestion de la SST; b) le processus d'apprentissage des compétences d'analyse des coûts-bénéfices basé sur des scénarios réels et aggravés ; c) l'identification et le calcul des coûts directs et indirects de SST.

\subsection{Résilience organisationnelle}

23

Selon plusieurs auteurs (Konninckx et Teneau, 2010; Dentz et Bailli, 2005; Rivest, 2010), la résilience organisationnelle est la capacité de rebondir et de reconstruire positivement l'entreprise lors d'événements ou lors d'un choc organisationnel. Elle varie d'une entreprise à l'autre selon le type d'événement (déclencheur ou accélérateur), les responsabilités qui incombent à l'entreprise (responsabilité envers les clients, le collectif de travail, l'état et l'environnement), les cadres d'analyse empruntés pour trouver une solution (unique vs multiple, habituel vs inhabituel, disciplinaire vs interdisciplinaire), ainsi que sa valeur de l'erreur (intégrer à la gestion quotidienne, annuelle et stratégique). La théorie de résilience est utile pour circonscrire les stratégies de résolution de problèmes face à des situations menaçantes, en faisant appel à des solutions inhabituelles, non usuelles pour l'entreprise ou le secteur de la production. Cette phase de reconstruction sera efficiente, si l'entreprise la perçoit comme une occasion d'apprentissage, de saisir des opportunités pour repenser à son identité, ses structures, ses modes de fonctionnement, ses réseaux de distribution, etc. (Koninckx et Teneau, 2010).

Cette reconstruction est une réponse à quatre types potentiels de menaces: 1) la menace identitaire de l'entreprise ; 2) la menace de survie ou de perte d'une part de marché ; 3) la menace de loyauté du collectif de travail, des clients, et d) la menace à la réputation de l'entreprise (Gravel et coll., 2013). La menace peut être unique ou multiple, les multiples menaces sont très souvent interactives. Selon leur capacité de résilience, les entreprises peuvent les nier, les banaliser, les prendre en considération comme une possibilité (calcul probabiliste) ou une éventualité (calcul de perte).

Les événements déclencheurs sont soudains, ils provoquent une onde de choc comme un accident de SST (Gravel et coll., 2013). L'événement latent est une situation connue, qui se répète sans gravité comme les troubles musculo-squelettiques (TMS), alors que l'événement accélérateur résulte de l'accumulation de situations plus ou moins appropriées, d'une aggravation d'une situation antérieurement tolérée comme l'augmentation des absences prolongées pour des TMS. Ces événements peuvent être internes, alors que d'autres peuvent être provoqués par des événements ou des personnes externes comme des inspections par des assureurs, des équipes de SST des sièges sociaux, etc.

La capacité d'analyse des solutions dépendra des avenues que l'entreprise adoptera en empruntant un cadre habituel comme l'analyse ergonomique ou l'analyse juridique ou en explorant un cadre inhabituel comme l'analyse psychodynamique des équipes de travail (Gravel et coll., 2013). Le présent projet incite les futurs gestionnaires (étudiants) à explorer toutes les solutions potentielles, des moins coûteuses aux plus 
dispendieuses, des plus économiques en matière d'efforts, de temps et d'argent, versus les plus rentables. Se servir de l'analyse des coûts-bénéfices pour établir ses critères de choix de solutions.

Figure 1. Résilience organisationnelle et la résolution de problèmes de santé et de sécurité au travail

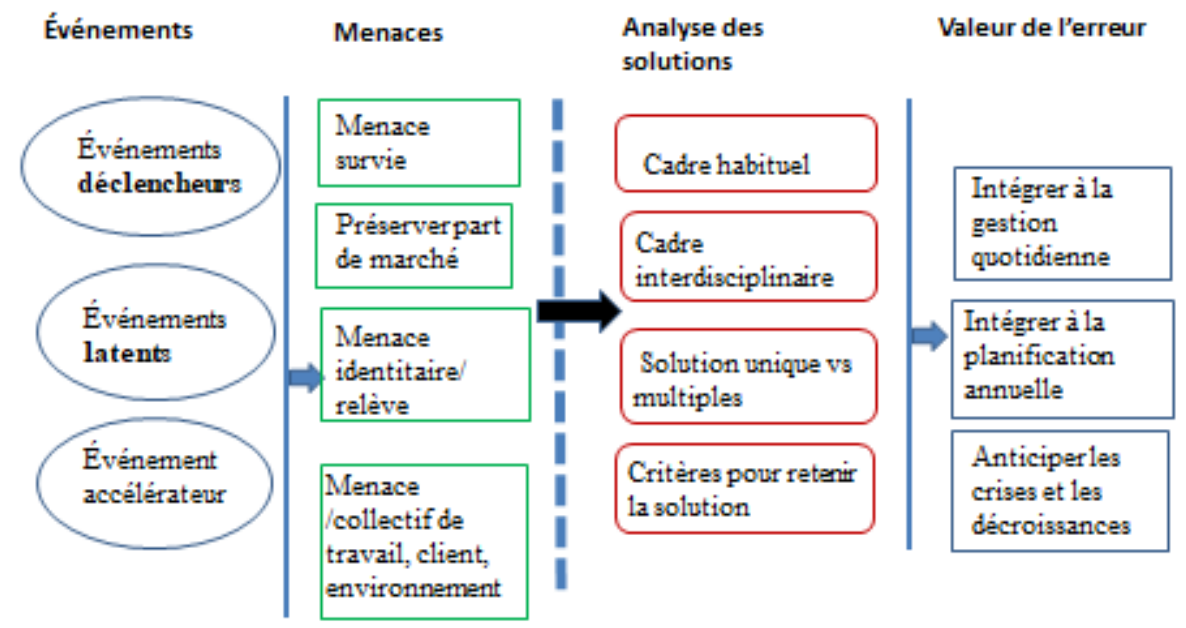

27 La dernière dimension, celle de la valeur de l'erreur, représente les moyens dont se dote l'entreprise pour s'assurer que ses capacités de résilience soient pérennes (Gravel et coll., 2013). Des mécanismes pour assurer que la rétroaction d'événements menaçants soit toujours présente en toutes circonstances, au sein de l'entreprise.

\subsection{Processus d'apprentissage coûts-bénéfices selon trois scénarios}

Pour documenter la capacité des gestionnaires en exercice et des futurs gestionnaires (étudiants) de nommer, de calculer, d'anticiper et d'estimer les coûts d'intervention selon divers scénarios, nous avons repris le modèle de Gravel et coll., (2013) en faisant varier les menaces, en partant de l'événement initial pour orienter la réflexion pour mener vers une situation aggravée et ultimement vers une menace. Le test d'apprentissage s'inscrit dans un crescendo de réactions d'une entreprise, qui témoigne de son niveau de résilience organisationnelle. Le test consistait à estimer les coûts directs et indirects pour trois scénarios d'une même situation :

a. une situation réelle, telle que rapportée dans un descriptif de cas liés à l'événement accidentel ou à la survenue des symptômes d'une maladie ;

b. une situation plus grave que la situation réelle, comme un accident pouvant avoir une atteinte plus sévère à la santé du travailleur victime de la situation ; 
c. une situation menaçante pour l'entreprise, alors que l'entreprise choisit de ne rien faire, et que cette décision entraîne, par exemple, une mobilisation des travailleurs faisant obstruction au cycle de production. différente d'examiner la construction d'un argumentaire efficient et crédible à soumettre aux instances supérieures de gestion des entreprises pour les convaincre d'investir dans la prévention. Cette démarche de construction de l'argumentaire repose essentiellement sur le caractère convivial de l'identification des coûts et des méthodes de calcul des ressources financières, matérielles et humaines comme le suggère le Centre patronal de santé et sécurité du travail du Québec (2005). Certes, dans un projet plus exhaustif, les critères de choix des interventions à retenir devraient inclure également la qualité préventive, les impacts, la stabilité, la faisabilité et les coûts de l'intervention (Bérubé, 2004). Ici, nous nous sommes modestement centrés sur l'analyse des coûts et non sur la qualité des interventions retenues.

\subsection{Identification et calcul des coûts directs et indirects de SST}

Comme l'illustre l'état de connaissance, la liste des coûts directs et indirects possibles, peu importe l'angle d'analyse empruntée, est exhaustive. Pour les fins de l'exercice de développer un outil d'apprentissage à la gestion des mesures préventives en santé et sécurité au travail (SST) basé sur les coûts, nous avons cru bon de réduire à sa plus simple expression cette liste de coûts: les coûts directs et indirects assumés par l'employeur.

\section{Coûts directs retenus}

Les coûts directs retenus sont ceux liés au traitement et à la réparation de la lésion (Lebeau et Duguay, 2011). Ils sont reliés à la lésion professionnelle comme les indemnités du travailleur blessé, son remplacement, les dommages matériels, les cotisations d'assurance et d'indemnisation, les frais médicaux, les coûts salariaux, les coûts liés aux pertes matérielles, les coûts administratifs, les coûts de production, les coûts commerciaux, les coûts de transport de l'accidenté, les coûts de poursuite judiciaire, etc. (Brody et coll., 1990 ; Lebeau et Duguay, 2011 ; Laroche, Dionne-Proulx et Legault, 2013).

\section{Coûts indirects retenus}

Les coûts indirects retenus sont les opportunités perdues par l'entreprise (Lebeau et Duguay, 2011). Ils incluent les coûts non assurés par l'assureur de l'entreprise, la formation des remplaçants, les heures supplémentaires des collègues, les pertes ou les baisses de productivité affectant les autres travailleurs ou à l'arrêt d'une machine, les dépenses de chauffage, d'électricité et de location, les coûts de remplacement et de réparation d'équipements, la valeur des dommages subis par la marchandise, les frais de nettoyage, la perte de contrat due à une perte d'expertise, mais aussi la baisse des cotisations à différents régimes gouvernementaux (assurance-emploi, assurance parentale, régime de rentes du Québec) et la diminution des recettes fiscales (Brody et coll., 1990 ; Tangri, 2003 ; Gosselin, 2004 ; Brun et Lamarche, 2006 ; Laroche et coll., 2013 ; Lebeau et Duguay, 2011). 
Tableau 1. Liste des coûts DIRECTS d'une lésion professionnelle retenus

\begin{tabular}{|c|c|c|}
\hline \multicolumn{3}{|l|}{ COÛTS DIRECTS } \\
\hline Coût & Définition & Employeur \\
\hline $\begin{array}{l}\text { Cotisations à la } \\
\text { CNESST }\end{array}$ & $\begin{array}{l}\text { Primes versées à la CNESST déterminées } \\
\text { selon la taille de l'entreprise et ses } \\
\text { performances antérieures }\end{array}$ & $\begin{array}{l}\text { Frais liés à l'hospitalisation, aux } \\
\text { traitements médicaux et à la } \\
\text { réadaptation } \\
\text { Indemnités de remplacement de } \\
\text { revenu, de décès, pour préjudice } \\
\text { corporel ou pour incapacité } \\
\text { permanente } \\
\text { Frais administratifs de la CNESST }\end{array}$ \\
\hline $\begin{array}{l}\text { Productivité lors } \\
\text { de l'accident }\end{array}$ & $\begin{array}{l}\text { Baisse de la productivité (arrêt, } \\
\text { ralentissement de production) due aux } \\
\text { dommages matériels ou aux accidents } \\
\text { affectant l'intégrité physique } \\
\text { Heures perdues par les employés }\end{array}$ & $\begin{array}{l}\text { Salaire des employés en arrêt ou } \\
\text { ralentissement de production } \\
\text { Salaire de l'employé accidenté } \\
\text { (journée de l'accident) }\end{array}$ \\
\hline $\begin{array}{l}\text { Frais } \\
\text { administratifs } \\
\text { directs }\end{array}$ & & $\begin{array}{l}\text { Salaire pour produire les rapports } \\
\text { d'enquête SST, formulaires CNESST } \\
\text { et autres formulaires }\end{array}$ \\
\hline Frais médicaux & $\begin{array}{l}\text { Dépenses engagées (ou anticipées) pour } \\
\text { soigner la lésion }\end{array}$ & $\begin{array}{l}\text { Salaire des secouristes ou infirmiers } \\
\text { au travail } \\
\text { Équipement de premiers soins } \\
\text { Frais de transport de l'accidenté } \\
\text { (autres que services d'urgence) }\end{array}$ \\
\hline $\begin{array}{l}\text { Services } \\
\text { d'urgence }\end{array}$ & $\begin{array}{l}\text { Tous services d'urgence pouvant } \\
\text { intervenir }\end{array}$ & Ambulances. \\
\hline $\begin{array}{l}\text { Dommages } \\
\text { matériels }\end{array}$ & $\begin{array}{l}\text { Tous dommages matériels survenus au } \\
\text { moment de la lésion }\end{array}$ & $\begin{array}{l}\text { Primes payées en assurance des } \\
\text { dommages matériels (attention aux } \\
\text { doubles comptes) } \\
\text { Frais de nettoyage } \\
\text { Perte de matières premières }\end{array}$ \\
\hline Frais funéraires & $\begin{array}{l}\text { Ensemble des frais pour inhumer un } \\
\text { travailleur décédé }\end{array}$ & $\begin{array}{l}\text { Frais funéraires indemnisés par la } \\
\text { CNESST }\end{array}$ \\
\hline
\end{tabular}

Tableau 2. Liste des coûts INDIRECTS d'une lésion professionnelle

\begin{tabular}{|l|l|l|}
\hline \multicolumn{2}{|l|}{ COÛTS INDIRECTS } \\
\hline Coût & Définition & Employeur \\
\hline
\end{tabular}




\begin{tabular}{|c|c|c|}
\hline Productivité & $\begin{array}{l}\text { Baisse de productivité (arrêt ou } \\
\text { ralentissement après l'accident) due aux } \\
\text { dommages matériels ou accidents } \\
\text { affectant l'intégrité } \\
\text { Heures de travail perdues par les } \\
\text { employés à cause de la suspension des } \\
\text { activités de production }\end{array}$ & $\begin{array}{l}\text { Heures perdues des travailleurs } \\
\text { dépendants et des superviseurs. } \\
\text { Baisse de productivité de l'accidenté } \\
\text { en assignation temporaire ou à son } \\
\text { retour } \\
\text { Baisse de productivité du } \\
\text { remplaçant } \\
\text { Productivité réduite due aux } \\
\text { dommages matériels. Pénalité de } \\
\text { retard de livraison }\end{array}$ \\
\hline Coûts salariaux & $\begin{array}{l}\text { Conséquences financières engendrées } \\
\text { par la variation du salaire de l'accidenté } \\
\text { et/ou des autres travailleurs } \\
\text { Heures de travail perdues par les } \\
\text { employés à cause de la suspension des } \\
\text { activités de production }\end{array}$ & $\begin{array}{l}\text { Primes pour heures supplémentaires } \\
\text { Hausse des salaires due à la hausse } \\
\text { du risque }\end{array}$ \\
\hline $\begin{array}{l}\text { Frais } \\
\text { administratifs } \\
\text { indirects }\end{array}$ & $\begin{array}{l}\text { Heures consacrées pour effectuer des } \\
\text { tâches administratives reliées à } \\
\text { l'accident. Frais généraux liés à } \\
\text { l'accident et à l'embauche d'un } \\
\text { remplaçant }\end{array}$ & $\begin{array}{l}\text { Frais administratifs de l'assureur } \\
\text { privé et public } \\
\text { Frais de recrutement/dotation } \\
\text { Frais de formation du remplaçant } \\
\text { Investigation de l'accident } \\
\text { Administration et suivi des dossiers }\end{array}$ \\
\hline Avantages sociaux & $\begin{array}{l}\text { Ensemble des avantages dont bénéficie } \\
\text { le salarié en sus du salaire }\end{array}$ & $\begin{array}{l}\text { Avantages sociaux assumés par } \\
\text { l'employeur, pour tout employé non } \\
\text { productif. }\end{array}$ \\
\hline $\begin{array}{l}\text { Travail ménager, } \\
\text { responsabilités } \\
\text { familiales }\end{array}$ & $\begin{array}{l}\text { Les services économiques perdus au } \\
\text { sein de la famille (extérieur du travail) }\end{array}$ & $\begin{array}{l}\text { Travail ménager indemnisé par la } \\
\text { CNESST }\end{array}$ \\
\hline Frais judiciaires & $\begin{array}{l}\text { Frais engendrés par des procédures } \\
\text { judiciaires }\end{array}$ & $\begin{array}{l}\text { Expertises médicales } \\
\text { Contestations médicales } \\
\text { Défenses de dossier } \\
\text { Poursuites }\end{array}$ \\
\hline Réputation & $\begin{array}{l}\text { Pertes financières reliées à l'image } \\
\text { négative qu'engendre un accident du } \\
\text { travail }\end{array}$ & $\begin{array}{l}\text { Pertes de contrats } \\
\text { Difficulté à recruter } \\
\text { Effets sur la réputation } \\
\text { (augmentation de frais marketing et } \\
\text { commerciaux) }\end{array}$ \\
\hline Autres frais & & $\begin{array}{l}\text { Autres frais non assurés } \\
\text { Chauffage/électricité, location } \\
\text { d'équipement à la suite de l'accident }\end{array}$ \\
\hline
\end{tabular}

33 Ces deux tableaux présentent une liste exhaustive de coûts pas nécessairement faciles à identifier parce qu'ils sont comptabilisés par différents départements: ressources humaines, entretiens, achats d'équipements et ventes. Des coûts qui peuvent aussi être 
comptabilisés en double, à la fois par les primes d'assurance et les cotisations à la CNESST. Enfin, une liste qui ne tient pas compte des coûts d'enquête et de prévention.

\subsection{Devis de recherche}

34 Cette étude phénoménologique est à la fois rétrospective (événement réel, passé) et prospective (situation aggravée, situation menaçante). La population à l'étude est composée de futurs gestionnaires. Le projet s'est déroulé en deux temps : 1) élaborer cinq études de cas avec des gestionnaires en exercice $(n=5)$ à partir d'un accident ou un épisode de maladie au sein de leur entreprise en se centrant sur les coûts directs et indirects engendrés par les événements; 2) tester les capacités des futurs gestionnaires, étudiants en maitrise $(n=22)$, à identifier les coûts des événements et à anticiper les coûts si les situations exposées s'aggravaient.

\subsection{Première étape : élaboration des cas}

35 La base de données est constituée de cinq cas d'entreprises de taille variable, du domaine privé et public, œuvrant dans la région de Montréal et dans divers secteurs d'activité économique. Pour chacune de ces cinq entreprises, nous avons interviewé le ou les gestionnaires en exercice concerné(s) par l'événement initial. Pour chacun de ces cinq événements, un descriptif de cas de 7 à 8 pages a été rédigé incluant une fine description de l'événement accidentel ou des circonstances de l'apparition des symptômes de la maladie des employés concernés, les efforts pour corriger ou redresser la situation, les heures dédiées à encadrer la situation, les dépenses engagées, les acteurs présents et absents dans la gestion de l'événement et la structure de gestion de la SST de l'entreprise (Sylvestre, 2017).

L'échantillon retenu est non probabiliste. Des huit entreprises sollicitées, cinq ont accepté de participer. La sollicitation s'est faite à partir du réseau de contacts des auteurs. Les cas ont été élaborés à partir d'entrevues semi-dirigées menées par les auteurs de cet article. Les entretiens ont été enregistrés avec le consentement des participants et retranscrits pour être transformés en récits de cas. Chaque entretien a duré en moyenne une heure et demie.

L'entreprise participante devait être capable de dresser une partie des coûts liés à l'événement de SST, pour un événement plus complexe qu'une simple chute sur la glace dans le stationnement de l'entreprise. L'événement devait s'être produit au cours des trois dernières années, et ce, même si le lien étiologique de la lésion avec l'emploi occupé par le plaignant pouvait être contesté ou ambigu comme dans le cas de violence au travail, des troubles neurologiques ou de dépendance. Les répondants étaient des gestionnaires occupant de hautes fonctions depuis quelques années au sein de l'entreprise, ils devaient pouvoir accéder aux données liées aux événements, aux coûts des lésions et de leurs réparations. Cette première étape a permis de répondre à la première question : quelles sont les composantes des coûts directs et indirects d'une lésion professionnelle que les gestionnaires en exercice peuvent identifier (compétence 1) et calculer facilement (compétences 2)? 


\subsection{Deuxième étape : tester l'outil d'apprentissage}

38 équipes) en gestion des ressources humaines inscrits à l'École de gestion de l'Université du Québec à Montréal au cours du semestre de l'automne 2016. Chaque équipe avait une semaine pour étudier chaque cas. Au terme de cette semaine, chaque équipe soumettait, au cours d'une séance de trois heures en classe, son analyse des coûts pour le cas, et ce, pour les trois scénarios : a) la situation réelle ; b) la situation aggravée et c) la situation présentant une menace. Les équipes comparaient les coûts qu'ils avaient identifiés, leurs méthodes de calcul et l'argumentaire qu'ils soumettraient aux décideurs, un argumentaire favorable ou non à un investissement en prévention. Pour la délibération finale, investir ou non en prévention, des rôles distincts étaient assignés aux différentes équipes comme dans un comité paritaire : la direction, le département ou l'équipe concernée, et les représentants des travailleurs.

Cet exercice a permis de répondre à la première question portant sur les compétences à identifier et calculer les coûts, mais surtout de répondre à la seconde question : quelles sont les composantes des coûts directs et indirects que les futurs gestionnaires (étudiants) sont capables d'anticiper (compétence 3) liés aux conséquences d'une approche non préventive? Si l'entreprise décide de ne rien faire et qu'une autre situation plus grave se produit (compétence 4) ou entraîne une menace pour le collectif de travail, les clients, leurs parts de marché ou leur réputation (compétence 4).

\subsection{Analyse des données}

Deux analyses ont été faites, l'analyse des coûts par l'étude de cas multiples et l'analyse transversale des compétences des étudiants, futurs gestionnaires. La première analyse a permis de proposer une grille simple et conviviale d'analyse des coûts. Et la seconde analyse a servi à identifier les coûts pertinents et utiles à l'élaboration d'un argumentaire pour la prise de décision. L'analyse des coûts des lésions professionnelles s'est basée sur la typologie des coûts directs et indirects. Ce qui a permis de répondre à la première question de recherche portant sur les compétences d'identifier et calculer les coûts. Alors que l'analyse transversale des compétences des futurs gestionnaires (étudiants) s'est appuyée sur les trois scénarios de résilience présentés dans le cadre d'analyse : la situation réelle, la situation aggravée et la situation menaçante pour l'entreprise. Apportant des éléments de réponses à la seconde question sur les compétences d'anticipation.

41 Les coûts présentés dans les cas sont estimés sur des valeurs datant de 2016-2017. Pour établir les coûts pour les situations aggravées et menaçantes, différents sites ont été consultés. Tous ces coûts sont discutables, voire contestables, mais l'essentiel de l'exercice est d'identifier les coûts et leur donner un ordre de grandeur si des situations aggravées et menaçantes se produisent. Le détail des montants et des calculs sont volontairement absents, parce que ces cas servent de matériel pédagogique.

\subsection{Résultats}

Les résultats sont présentés en deux sections. La première section présente l'analyse des coûts par cas, incluant un bref descriptif de l'entreprise, de l'événement et des 
coûts. Une analyse centrée sur la capacité de circonscrire (compétence 1) et de calculer les coûts (compétence 2), analyse à laquelle les gestionnaires en exercice et les étudiants en gestion ont participé. La seconde analyse, l'analyse transversale, est centrée sur les compétences à anticiper les conséquences d'une approche non préventive (compétence 3) et à estimer les coûts des situations plus graves se produisant (compétence 4) ou entraînant une menace pour le collectif de travail, les clients, leurs parts de marché ou leur réputation (compétence 4). Pour cette seconde analyse, nous n'avons que les observations des étudiants qui se sont soumis à l'exercice de l'outil d'apprentissage, aux études de cas et aux discussions en classe.

\section{Analyse des coûts par cas}

Dans le tableau suivant, les événements, les coûts et les menaces pour les cinq cas à l'étude sont présentés sommairement. Tous les cas sont réels, mais afin de préserver l'anonymat de l'entreprise et des gestionnaires, de nouveaux noms leur ont été attribués et leurs caractéristiques ont été légèrement modifiées. Toutes associations d'idées avec une entreprise précise seraient le fruit du hasard.

Les situations aggravées et menaçantes ici retenues pour illustrer l'analyse de coûts sont celles les plus fréquemment nommées par les étudiants, des situations crédibles pour construire un argumentaire et convaincre une équipe de décideurs d'investir en prévention. Certes les coûts sont approximatifs et possiblement modifiables selon d'autres références que celles que nous avons utilisées. Le but étant de centrer l'exercice sur des scénarios de récidives, si l'entreprise adopte la posture de ne rien faire.

Tableau 3. Synthèse des analyses de coûts pour l'ensemble des cas à l'étude selon les situations réelles, aggravées et menaçantes

\begin{tabular}{|c|c|c|c|c|c|}
\hline Cas & $\begin{array}{l}\text { Bibliothèque } \\
\text { du génie }\end{array}$ & Long repos & $\begin{array}{l}\text { Aux Grands } \\
\text { Manèges }\end{array}$ & $\begin{array}{l}\text { Grands } \\
\text { Bâtiments }\end{array}$ & $\begin{array}{l}\text { Aux } \\
\text { Chaudrons } \\
\text { des pauvres }\end{array}$ \\
\hline Secteur & $\begin{array}{l}\text { Services } \\
\text { culturels }\end{array}$ & $\begin{array}{l}\text { Hébergement } \\
\text { longue durée }\end{array}$ & Loisirs & Construction & $\begin{array}{l}\text { Aide } \\
\text { alimentaire }\end{array}$ \\
\hline Situation réelle & $\begin{array}{l}\text { Technicien } \\
\text { bibliothécaire } \\
\text { Maladie } \\
\text { mentale } \\
\text { d'origine } \\
\text { neurologique : } \\
\mathbf{1 3 4} 250 \text { \$: } \\
43 \% \\
\text { dommage } \\
57 \% \\
\text { correction }\end{array}$ & $\begin{array}{l}\text { Préposée (1) } \\
\text { aux } \\
\text { bénéficiaires } \\
\text { Plaintes pour } \\
\text { harcèlement : } \\
\mathbf{2 6 2} 400 \text { \$ : } \\
8 \% \text { dommage } \\
92 \% \\
\text { correction }\end{array}$ & $\begin{array}{l}\text { Choc post- } \\
\text { traumatique } \\
\text { d'un commis } \\
\text { à l'admission } \\
\text { à la suite du } \\
\text { vol à main } \\
\text { armée : } \\
460250 \$: \\
4 \% \\
\text { dommage } \\
96 \% \\
\text { correction }\end{array}$ & $\begin{array}{l}\text { Brûlure } \\
\text { chimique à } \\
\text { d'un } \\
\text { technicien en } \\
\text { bâtiment : } \\
59800 \text { \$ : } \\
69 \% \\
\text { dommage } \\
31 \% \\
\text { correction }\end{array}$ & $\begin{array}{l}\text { Dépression, } \\
\text { dépendance } \\
\text { aux jeux et à } \\
\text { l'alcool chez } \\
\text { le directeur: } \\
850 \$ \text { : } \\
100 \% \\
\text { dommage }\end{array}$ \\
\hline
\end{tabular}




\begin{tabular}{|c|c|c|c|c|c|}
\hline Situation aggravée & $\begin{array}{l}\text { Cadre affecté } \\
\text { par le même } \\
\text { type de } \\
\text { maladie } \\
\text { Situation } \\
\text { réelle } \\
\text { aggravation } \\
\mathbf{1 1 6} 700 \text { \$) : } \\
96 \% \\
\text { dommage } \\
4 \% \\
\text { correction }\end{array}$ & 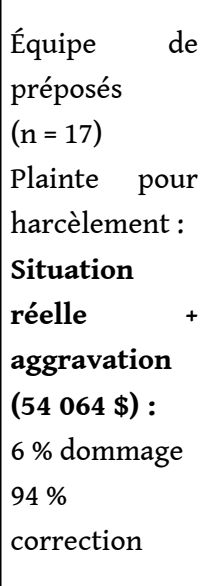 & $\begin{array}{l}\text { Même } \\
\text { employé } \\
\text { mais blessé } \\
\text { par balle : } \\
\text { Situation } \\
\text { réelle + } \\
\text { aggravation } \\
(49750 \text { \$) : } \\
14 \% \\
\text { dommage } \\
86 \% \\
\text { correction }\end{array}$ & 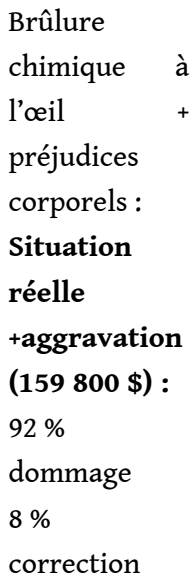 & $\begin{array}{l}\text { Rechute du } \\
\text { directeur } \\
\text { après } 1 \mathrm{e} \\
\text { première } \\
\text { thérapie : } \\
\text { Situation } \\
\text { réelle + } \\
\text { aggravation } \\
(\mathbf{1 1} 200 \text { \$): } \\
100 \% \\
\text { dommage }\end{array}$ \\
\hline $\begin{array}{l}\text { Situation } \\
\text { menaçante }\end{array}$ & $\begin{array}{l}\text { Grief syndical } \\
\text { réintégration } \\
\text { de l'employé } \\
\text { / poste : } \\
\text { Dommage } \\
\text { initial } \\
\text { menace } \\
(51550 \text { \$) = } \\
97195 \$ \\
\text { dommage vs } \\
76522 \text { \$ } \\
\text { correction }\end{array}$ & $\begin{array}{l}\text { Démission } \\
30 \% \text { des } \\
\text { employés : } \\
\text { Dommage } \\
\text { initial } \\
\text { menace } \\
(433530 \text { \$) = } \\
454250 \text { \$ } \\
\text { dommage vs } \\
241408 \text { \$ } \\
\text { correction }\end{array}$ & $\begin{array}{l}\text { Refus de } \\
\text { travail des } \\
\text { employés : } \\
\text { Dommage } \\
\text { initial } \\
\text { menace } \\
(193650 \text { \$) = } \\
212060 \text { \$ } \\
\text { dommage vs } \\
441840 \text { \$ } \\
\text { correction }\end{array}$ & $\begin{array}{l}\text { Refus de } \\
\text { travail des } \\
\text { employés : } \\
\text { Dommage } \\
\text { initial } \\
\text { menace } \\
(132900 \text { \$) = } \\
174106 \text { \$ } \\
\text { dommage vs } \\
18540 \text { \$ } \\
\text { correction }\end{array}$ & 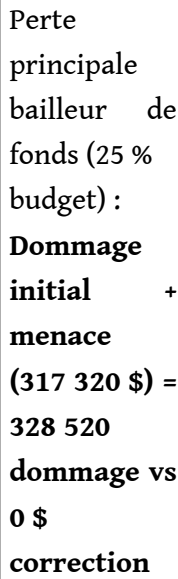 \\
\hline
\end{tabular}

\subsection{Bibliothèque du génie}

La Bibliothèque du génie est une bibliothèque d'une petite municipalité qui emploie 27 salariés syndiqués à temps plein et à temps partiel : bibliothécaires, techniciens à la documentation, aides à la documentation et le directeur. Un des techniciens à la documentation commet de nombreuses erreurs de travail, obligeant un autre technicien à revoir systématiquement son travail. Son rendement était inadéquat, il n'effectuait que $20 \%$ de ses tâches, et ce, avec 50 \% d'erreurs de classement des livres. Il s'est avéré que ce technicien souffrait d'épilepsie sans limitation fonctionnelle. À la suite d'une crise d'épilepsie, l'obligeant à être hospitalisé pendant trois mois, après sa convalescence son travail n'était pas à la hauteur. Après une année de supervision et d'accommodement, les délégués syndicaux et la direction ont convenu de rétrograder ce technicien à un poste d'aide à la documentation, au lieu de le congédier. Le nombre d'heures de travail a été augmenté, lui permettant ainsi de recevoir un revenu équivalent à celui d'un technicien à temps partiel. Pendant quatorze mois, le directeur de la bibliothèque et la bibliothécaire ont supervisé et évalué les erreurs de l'employé peu performant.

Les étudiants ont eu du mal à distinguer les coûts directs et indirects, les informations et les coûts rapportés par le gestionnaire de l'entreprise n'étaient pas toujours bien circonscrits. Malgré cette lacune, ils ont quand même réussi à établir correctement 
l'ensemble des coûts liés à la perte de productivité : le temps des autres employés pour corriger les erreurs du technicien, le temps de supervision du supérieur immédiat, les rencontres syndicales et le temps d'encadrement du processus d'évaluation par le directeur. La somme des coûts de la situation réelle est estimée à 134250 \$ selon les échelles salariales en vigueur au moment des événements. Si la situation avait affecté un cadre la situation aggravée aurait coûté $116700 \$$ de plus à cause du salaire supérieur. Par contre, si l'entreprise n'avait rien fait, aucune correction et que les employés s'étaient mobilisés contre l'employeur, la menace d'un arrêt de travail aurait coûté 51550 \$ de plus que les dommages initiaux.

D'autres observations sont importantes à souligner. D'abord, les étudiants ont confondu lésion psychologique d'origine professionnelle et maladie mentale liée à une condition personnelle antérieure, ce qui augmentait de façon importante des coûts imputables à l'entreprise. Seconde observation, la présence syndicale a joué un rôle important dans le scénario d'une situation menaçante. Les étudiants ont su mobiliser leurs habiletés en relations de travail pour scénariser une situation impliquant le dépôt d'un grief syndical. Deux situations d'apprentissage importantes dans le développement des compétences pour circonscrire et calculer les coûts, et les menaces.

\subsection{Au Long Repos}

Au Long Repos est un établissement de soins de longue durée pour personnes en perte d'autonomie. Cet établissement est la résultante de la fusion de quatre établissements du réseau de la santé. Cette nouvelle structure a réduit l'encadrement à une seule équipe de direction engendrant une perte identitaire, des frustrations, des hostilités, menant à une plainte pour harcèlement psychologique, des accusations jugées, après enquête, non recevables. L'enquête a aussi révélé que les employés mis en accusation avaient manqué de respect envers les patients. La direction des ressources humaines s'est interrogée à la fois sur l'application du code déontologique de l'établissement et les pratiques éthiques de son personnel envers les patients, et le silence des parents des patients en regard des agissements du personnel. Des circonstances menant l'équipe de direction à revoir toutes ses pratiques de gestion incluant : les effectifs d'encadrement, le climat de travail, les horaires de travail, la permanence des postes et les problèmes de recrutement. Une démarche qui s'est étalée sur près d'une année, et qui a mobilisé plus de 200 employés à participer à des ateliers de perfectionnement.

Les coûts de l'événement sont principalement associés à la perte de productivité et aux frais administratifs: le temps des préposés aux soins dédiés aux enquêtes, le remplacement des employés suspendus, la diminution de $25 \%$ de la performance de la victime, le temps du conseiller en ressources humaines, les honoraires du conseiller en éthique. L'estimation des coûts totaux pour l'enquête sur le harcèlement psychologique est de 262450 \$ pour l'employeur. Si la situation de harcèlement et la dépression qui s'en est suivie avaient été reconnues comme découlant du travail, ce scénario d'une situation aggravée est estimé à 316500 \$. Une situation compromettant l'image de l'entreprise au regard de son personnel et ses bénéficiaires, pouvant entraîner des poursuites judiciaires et la fermeture de plusieurs lits, une situation menaçante estimée à 695950 \$, soit deux fois et demie le coût de l'événement.

Il s'agissait du deuxième cas analysé, un cas complexe pour lequel les futurs gestionnaires (étudiants) avaient peu d'expérience d'analyse de situations aggravées, 
donc peu de compétences spécifiques. Bien que les conséquences du harcèlement aient été bien identifiées, étonnamment, il a été nécessaire de rappeler les coûts des conséquences aux manquements éthiques des employés envers la clientèle. Un apprentissage important pour de futurs gestionnaires en ressources humaines et responsables de SST confrontés aux situations de harcèlement au travail et de problèmes de santé mentale.

\subsection{Aux Grands Manèges}

51 Aux Grands Manèges est une entreprise récréotouristique saisonnière, qui embauche l'équivalent de 57 personnes à temps plein pendant six mois, soit environ 180 jours. L'entreprise compte plusieurs postes: administration, électromécanique, entretien sanitaire et des bâtiments, billetterie, préposé aux manèges et à la restauration.

Un jour de grand achalandage, un vol à main armée ${ }^{3}$ a été perpétré à la billetterie. Personne n'a été blessé, ni employé, ni client, mais l'entreprise a dû suspendre ses entrées pendant cinq heures. L'employé victime de l'agression a subi un choc posttraumatique ayant pour conséquence: dix jours de congé de maladie, une affectation temporaire d'un mois à un poste à l'administration générale et une consultation hebdomadairement d'une psychologue du programme d'aide aux employés (P.A.E.) pendant six mois. Dans les jours qui ont suivi, le personnel a reçu une aide psychologique. L'entreprise a aussi entrepris des démarches afin de corriger la situation : a) rendre les guichets sécuritaires ; b) mieux analyser les volumes de flux des entrées; c) installer des caméras de surveillance; d) former tous les employés sur les vols à main armée. En considérant les coûts directs liés à la perte de productivité, aux frais médicaux et à l'intervention immédiate d'un psychologue, les coûts de l'événement sont estimés à 28370 \$. Les coûts de correction impliquant les modifications d'équipements (guichets, caméras), l'analyse des flux de clientèle et la formation du personnel sur les agressions armées sont estimés à $431880 \$$, soit $96 \%$ des coûts liés à l'événement et pour lequel l'entreprise a tout de suite réagi. Des coûts de correction quinze fois supérieurs aux coûts d'un événement.

Si l'entreprise avait pris la décision de ne rien faire, en misant sur le fait que l'événement était exceptionnel, qu'il ne s'était jamais produit auparavant, mais qu'un même incident se produit avec, cette fois, l'employé qui est atteint par une balle à un membre supérieur, on peut facilement ajouter $80000 \$$ aux coûts directs de l'événement. Malgré cela, il aurait été moins coûteux de ne rien faire que d'implanter tous les correctifs que l'entreprise a ici retenus incluant les frais indirects ( 431880 \$) liés à l'événement.

Dans l'exercice, les étudiants ont facilement anticipé les conséquences d'une agression armée, si l'entreprise avait dû fermer le site pour une journée complète parce que les employés et leurs syndicats exerçaient un droit de refus de travail, provoquant une mauvaise image de l'entreprise au sein du public, l'image d'un lieu non sûr pour leur famille. Les coûts de cette situation menaçante ont été estimés à 193640 \$. Plus expérimentés par l'analyse des cas précédents, les étudiants ont mieux cerné les différents coûts directs et indirects. Par contre, ils ont été déstabilisés face à un risque qui appartient aux milieux bancaires et aux commerces de détail, alors qu'il s'agissait d'un cas d'un lieu de divertissements. Soulignons également que les étudiants étaient plus préoccupés par une deuxième agression armée, que par la menace d'une action 
syndicale. Cet exercice leur a fait prendre conscience des possibles dissonances entre la vocation de l'entreprise et les risques potentiels associés au secteur d'activité économique.

\subsection{Grands Bâtiments}

55 Grands Bâtiments est une entreprise de la construction et de génie civil qui emploie 85 travailleurs sur les chantiers, 30 personnes à l'administration, 20 camionneurs et cinq employés à l'atelier mécanique, affiliés à des sections syndicales selon leur métier. Lors d'un travail d'excavation pour colmater une brèche dans un égout, un travailleur a utilisé un mélange à ciment et de colle ayant la propriété de durcir rapidement. Un produit d'usage courant, qui ne provoque pas d'émanations toxiques, mais au contact de la peau, devient un puissant irritant. Après avoir colmaté la fissure, le travailleur a ressenti une brûlure, sans dommage apparent à son vêtement. Le médecin consulté diagnostique une brûlure thermique, alors qu'il s'agissait d'une brûlure chimique. Une erreur du diagnostic menant à un traitement inapproprié, qui a entraîné une aggravation de la plaie nécessitant une greffe de la peau. La situation a incité l'entreprise à constater plusieurs lacunes dans notre système de soins et de santé pour identifier l'agresseur chimique et traiter ces cas de brûlures chimiques, et dans le Système d'information sur les matières dangereuses utilisées au travail (SIMDUT) (système général harmonisé). Le préventionniste de l'entreprise a pris l'initiative de développer un programme de prévention comprenant l'achat du produit neutralisant les brûlures chimiques, la diphotérine ${ }^{4}$, la formation des employés œuvrant sur les chantiers de l'entreprise et ceux de toutes entreprises du secteur de la construction utilisatrices de tels produits chimiques.

L'événement accidentel, considérant la perte de productivité, les frais médicaux et le replacement du travailleur blessé, n'a coûté que 10000 \$, alors que le travailleur a bénéficié d'une assignation temporaire pour l'équivalent de $80 \%$ du temps de sa période de rétablissement. Les coûts de correction impliquant une formation sur l'intervention d'urgence ( 85 employés) et l'achat des trousses du produit neutralisant (600\$/trousse $\times 20$ chantiers) sont estimés à $32100 \$$.

57 Pour ce quatrième cas, l'expérience a fait son œuvre, les étudiants ont mieux circonscrit et calculé les coûts directs et indirects. Néanmoins, ils avaient encore de la difficulté à imaginer une situation aggravée, comme une brûlure touchant un organe sensible tel que l'œil (estimé à 100000 \$ en coûts supplémentaires). Par contre, ils se sont révélés très habiles à scénariser une situation menaçante provenant du collectif de travail comme la menace d'une action syndicale, une grève de quelques jours (estimée à 75000 \$ incluant des pénalités pour non-respect des délais de livraison des travaux). Soulignons qu'ils ont été étonnés par le leadership du préventionniste pour développer une formation pour prévenir d'autres lésions au sein de l'entreprise et auprès de tout le secteur de la construction. Néanmoins, ils ont pu mesurer les bénéfices de visibilité d'investir dans le développement de mesures préventives, surtout si ces actions rapportent une reconnaissance de la part des entreprises œuvrant dans le même secteur d'activité. 


\subsection{Aux Chaudrons des pauvres}

58 alimentaire des personnes en situation d'extrême pauvreté. Cet organisme sans but lucratif (OSBL) sert quotidiennement 350 dîners cinq jours par semaine, et distribue mensuellement 750 paniers de provisions. Mis à part le directeur général, l'équipe comprend huit employés à l'administration et à la cuisine. Issue d'une tradition philanthropique, l'entreprise fonctionne avec un budget de 1,2 million de dollars provenant des fonds publics, de dons privés d'argent et des dons de nourriture offerts par les épiceries et les établissements du quartier. Au cours de la dernière année, l'établissement a élargi sa mission en distribuant annuellement 200000 repas dans les écoles et les résidences pour aînés du quartier. Neuf nouvelles personnes ont été engagées pour mener à bien ce nouveau service. Le directeur, ravi de relever un nouveau défi, a sous-estimé l'ampleur de la tâche et pour masquer sa solitude, il s'est tourné vers l'alcool et le jeu pour combler son désarroi affectif. Au début, il consommait occasionnellement. Mais avec le temps, il a dû emprunter à la petite caisse et la carte de crédit de l'entreprise pour couvrir ses dépenses personnelles. Au terme d'une année difficile, le directeur a été victime d'un problème de santé sévère, l'empêchant de travailler pendant deux mois. À cause de sa santé précaire, il a oublié de préparer la demande de renouvellement de la subvention adressée au plus important bailleur de fonds. Inquiète, l'équipe a fortement réagi à l'insécurité financière de l'organisation en faisant les démarches de syndicalisation. Malgré les nombreuses et infructueuses propositions d'accommodement auprès de son directeur en détresse et l'insécurité grandissante des employés, le C.A. a procédé au congédiement du directeur.

$\mathrm{Au}$ terme de cette cinquième étude de cas, les étudiants ont facilement identifié et départagé les coûts directs et indirects, d'administration, d'encadrement, de perte de productivité et une indemnité de départ. Un coût d'événement estimé à 17000 \$, une somme insignifiante comparativement aux coûts engendrés par la situation aggravée par l'insécurité des employés qui ont choisi de se syndiquer. Cette démarche de syndicalisation a été estimée à $62400 \$$. Bien que la syndicalisation engage des dépenses, elle ne constituait pas une menace. Par contre, les délais pour soumettre la demande de renouvellement de la subvention, une subvention établie à 317320,00 \$, soit $25 \%$ du budget de fonctionnement de l'organisation, un délai qui constituait une menace évidente pour les étudiants. Avec ce cinquième exercice, les étudiants avaient acquis des aptitudes à anticiper et comptabiliser les coûts des situations menaçantes crédibles afin de convaincre la direction des entreprises d'intervenir en SST.

\section{Construire un argumentaire convaincant pour investir en SST}

60 À la lumière de nos résultats nous constatons que pour construire habilement un argumentaire convainquant afin d'investir en SST, les gestionnaires doivent: a) identifier, classer et chiffrer les coûts et b) développer des scénarios plausibles de situations aggravées ou menaçantes. Toutefois, lors du premier cas, celui de la Bibliothèque du génie, nous avons constaté la difficulté des futurs gestionnaires (étudiants) à distinguer ce qui est lié au travail de ce qui est une condition personnelle.

Perspectives interdisciplinaires sur le travail et la santé, 22-1 | 2020 
61 La distinction de ce qui est lié au travail de ce qui est une condition personnelle est essentielle pour analyser correctement un événement SST et les coûts qui lui sont attachés. Une distinction complexe lorsqu'il s'agit de problème de santé mentale, comme dans les cas de la Bibliothèque du génie et Aux Chaudrons des pauvres. Le premier étant une condition personnelle, une maladie neurologique sans lien étiologique avec le travail, alors que dans le deuxième cas la détresse psychologique a été engendrée et aggravée par une sous-estimation des compétences et du temps requis pour réaliser adéquatement une nouvelle tâche. Des situations qui n'ont pas les mêmes conséquences sur les coûts d'indemnisations réclamées à la CNESST, à l'assurance collective ou l'assurance-emploi. En fait, parmi tous les calculs de coûts, les plus difficiles à classer, identifier et anticiper, ce sont les coûts liés aux conséquences de problèmes de santé mentale.

La capacité d'identifier, classer et chiffrer correctement les coûts d'un événement de SST est à la base de l'élaboration de l'argumentaire adressé à la direction d'une entreprise pour la prise de décision d'intervenir ou non en matière de SST. Les compétences à circonscrire et calculer les coûts s'acquièrent rapidement, après l'apprentissage de trois cas. Les coûts directs et indirects sont facilement circonscrits, bien qu'ils puissent être confondus les uns avec les autres, surtout avec les coûts de réparation lorsqu'ils sont appliqués immédiatement lors de l'événement comme dans le cas des Grands Manèges. Les coûts directs facilement identifiables sont: les pertes de revenus, les coûts de remplacement des absents (recrutement, formation, supervision) et les coûts d'intervention d'urgence. Les coûts indirects de l'événement qui reviennent le plus facilement sont les hausses de coûts de cotisation, les libérations syndicales pour procéder au processus de résolution du problème, les frais pour freiner ou faire obstacle à une menace pouvant ternir l'image de l'entreprise, et les coûts liés à une poursuite judiciaire.

Il demeure que l'exercice de distinguer les coûts d'événement et les coûts de réparation est laborieux pour plusieurs. Il est pourtant essentiel pour nuancer les coûts associés aux scénarios entre ne rien faire, subir les conséquences d'une situation aggravée ou menaçante. Il aurait aussi été intéressant d'inclure dans le processus d'apprentissage, l'analyse des coûts des corrections durables.

64 Les étudiants ont généralement bien circonscrit les situations aggravées comportant des risques de limitations fonctionnelles ou de handicaps. Ils ont aussi très bien saisi les conséquences de menaces potentielles, entre autres, les menaces de grève des employés, de griefs syndicaux, les poursuites juridiques, le mécontentement des clients lésés et l'image de marque ternie. Dans le cas Grands Bâtiments, certaines équipes d'étudiants ont également identifié des menaces environnementales en cas de déversements chimiques, sans toutefois souligner la nécessité de revoir l'implantation du SIMDUT. Ces menaces sont transformées avec aisance en arguments pour aider le processus de décision, à savoir apporter des correctifs ou maintenir le statu quo.

$\mathrm{Au}$ terme de ces études de cas centrées sur les analyses de coûts, les étudiants avaient l'impression d'avoir franchi un désert de chiffres tout en acquérant une maturité intellectuelle: construire un argumentaire basé sur des données et non sur des impressions, un argumentaire qui s'inscrit dans le raisonnement logique des décideurs, des directeurs de département et des conseils d'administration comme le mentionne Laufer (1987). 


\section{Discussion et conclusion}

Il a été possible, à partir de la littérature, de constituer une grille qui compile l'ensemble des coûts directs et indirects, une grille plus simple, moins exhaustive que le « Health \& Safety Smart Planner » (Brun et Lamarche 2004 ; Brody et coll., 1990 ; Lebeau et Duguay, 2011 ; Gosselin, 2004 ; Laroche et coll., 2013 ; Tangri, 2003). Selon plusieurs auteurs, même si l'identification et le calcul des coûts sont achevés, l'évaluation économique d'une lésion professionnelle qui compare les coûts et les conséquences des choix entre ne rien faire et intervenir est rarement faite (Organisation mondiale de la santé, 2003 ; Drummond et coll., 2005 ; Hoch et Dewa, 2005 ; Lejeune, 2008 ; Brouselle et coll., 2009 ; Uegaki et coll., 2010 ; Sultan-Taïeb, 2011).

Dans le présent projet, nous avons volontairement limité nos observations à l'identification et au calcul des coûts des événements et des situations aggravées et menaçantes. Le projet ne reposait pas sur les prémisses des économistes de coûtsefficacités ou coûts-bénéfices de choisir une intervention ou un programme le plus efficient parmi plusieurs options. Le projet était orienté sur le processus décisionnel des gestionnaires, choisir entre deux options, investir dans la prévention ou maintenir le statu quo jusqu'au prochain événement. Cette démarche d'apprentissage est originale puisque dans la littérature le processus décisionnel est rarement abordé de cette façon. Si l'évaluation des coûts constitue un bon argumentaire pour orienter et convaincre les gestionnaires d'investir en SST, les outils d'analyse sont rares et peu conviviaux (Brun et Lamarche, 2006 ; Sultan-Taïeb, 2011 ; Dietrich, 1999 ; Baril-Gingras et coll., 2010).

8 Les résultats des analyses des cinq cas ont confirmé les composantes importantes des coûts directs et indirects identifiées dans la littérature. Ils ont également souligné l'importance pour les gestionnaires en exercice et les futurs gestionnaires (étudiants) de deux éléments de coûts sous-estimés par la littérature : ceux liés à la libération syndicale et ceux liés aux avantages sociaux. L'analyse des études de cas a démontré l'apport du syndicat, pas nécessairement dans l'analyse des coûts directs et indirects, mais dans l'anticipation des situations aggravées ou menaçantes pouvant mener à l'inflation des coûts si l'entreprise préfère ne rien faire. Finalement, nous avons également constaté que les avantages sociaux payables par l'employeur sont facilement oubliés par les étudiants lorsqu'un salaire est comptabilisé.

Nous avons résumé les coûts utiles et facilement identifiables dans le cadre d'un processus d'apprentissage, dans la Grille d'analyse des coûts d'une lésion selon les situations réelle, aggravée, menaçante (Tableau 4). Soulignons que l'importance de ces composantes peut varier selon la taille de l'entreprise, le contexte organisationnel et les menaces pouvant nuire à l'entreprise. Ce qui constitue une des limites du projet qui repose sur l'étude de cinq entreprises, de secteurs d'activité et de taille fort variables

L'analyse des situations aggravées a démontré qu'au début les étudiants peinaient à anticiper des situations aggravées ou menaçantes pour soutenir leur argumentaire favorable au déploiement de mesures préventives de SST. Une difficulté que nous avons associée à la tendance des gestionnaires à dédier leurs efforts à éliminer les coûts directs des lésions professionnelles, sous-estimant les coûts indirects et délaissant les coûts engendrés par des menaces à long terme (Cadieux et coll., 2004; Roy, Cadieux et Desmarais, 2005; Champoux et Brun, 2010; Vinet, 2015). De plus, n'ayant pas nécessairement de connaissances comptables pour les calculs complexes, les prévisions 
fictives et les comparaisons abstraites des coûts, l'exercice d'anticiper les coûts de SST pour des situations aggravées ou menaçantes peut décourager et démotiver les gestionnaires aux prises avec de multiples tâches de gestion liées aux opérations, à la qualité, aux ressources humaines et à la SST. Néanmoins, nous avons observé, chez les étudiants, qu'ils peuvent acquérir rapidement et apprécier cette compétence, mais que les compétences à identifier et à calculer les coûts directs et indirects sont conditionnées par le scénario dans lequel l'exercice est fait. Les coûts directs sont plus facilement identifiés lorsqu'il s'agit d'analyser des situations réelles, alors que les coûts indirects liés aux frais médicaux, aux indemnités de remplacement et aux assurances sont mieux circonscrits lors des situations aggravées. Finalement, notons que tous les frais juridiques, de libérations syndicales et de représentations médiatiques sont plus précis lorsqu'il s'agit d'analyser les coûts de situations menaçantes. Il semblerait que les futurs gestionnaires (étudiants) développent rapidement des compétences à anticiper des situations aggravées et menaçantes, des éléments convainquant pour élaborer un argumentaire favorable à l'investissement préventif à adresser à une équipe de direction ou un conseil d'administration. Toutefois, il faut que cet apprentissage se fasse avant que les événements tragiques se produisent, qu'il s'acquière par simulation, développer une résilience proactive et non pas seulement réactive (Cadieux et Pérusse, 2013). Il faut décourager la stratégie de gestion réactive de la SST. Le tableau 4 fait une synthèse de ces habiletés qui se sont accrues avec la progression des études de cas.

Tableau 4. Grille d'analyse des coûts d'une lésion selon les situations réelles, aggravées, menaçantes

\begin{tabular}{|c|c|c|c|c|}
\hline \multicolumn{2}{|l|}{ COÛTS DIRECTS } & & \multirow{2}{*}{\begin{tabular}{l|} 
Situations \\
aggravées
\end{tabular}} & \\
\hline & & réelles & & menaçantes \\
\hline $\begin{array}{l}\text { Cotisations à la } \\
\text { CNESST }\end{array}$ & $\begin{array}{l}\text { Augmentation des cotisations à la CNESST, } \\
\text { frais d'assistance médicale et de réadaptation, } \\
\text { indemnités de remplacement de revenu, de } \\
\text { décès, préjudice corporel, incapacité } \\
\text { permanente, amendes CNESST. }\end{array}$ & & v & $\checkmark$ \\
\hline $\begin{array}{l}\text { Productivité } \\
\text { lors de } \\
\text { l'accident }\end{array}$ & $\begin{array}{l}\text { Salaire accidenté et employé en arrêt de } \\
\text { travail ou ralentissement de production, } \\
\text { journée de l'accident + avantages sociaux. }\end{array}$ & $\sqrt{ }$ & v & $\checkmark$ \\
\hline $\begin{array}{l}\text { Frais } \\
\text { administratifs } \\
\text { directs }\end{array}$ & $\begin{array}{l}\text { Salaire pour produire les rapports d'enquête } \\
\text { SST, formulaires CNESST et autres formulaires } \\
\text { + avantages sociaux. }\end{array}$ & $\sqrt{ }$ & v & \\
\hline Frais médicaux & $\begin{array}{l}\text { Salaire des secouristes ou infirmiers au travail } \\
\text { + avantages sociaux, trousse de premiers soins, } \\
\text { frais de transport de l'accidenté (autres que } \\
\text { services d'urgence), ambulances. }\end{array}$ & & v & $\checkmark$ \\
\hline
\end{tabular}




\begin{tabular}{|c|c|c|c|c|}
\hline $\begin{array}{l}\text { Dommages } \\
\text { matériels }\end{array}$ & $\begin{array}{l}\text { Réparations ou remplacement sur machinerie } \\
\text { et équipement, dommages causés à la } \\
\text { marchandise, frais de nettoyage, perte de } \\
\text { matières premières. }\end{array}$ & V & v & $\sqrt{ }$ \\
\hline Autres frais & $\begin{array}{l}\text { Augmentation des cotisations aux assurances, } \\
\text { poursuites criminelles. }\end{array}$ & & v & \\
\hline \multicolumn{2}{|l|}{ COÛTS INDIRECTS } & & Situation & \\
\hline & & réelle & aggravée & menaçante \\
\hline $\begin{array}{l}\text { Productivité à } \\
\text { long terme }\end{array}$ & $\begin{array}{l}\text { Heures perdues et baisse de productivité } \\
\text { (travailleurs dépendants/superviseurs, } \\
\text { accidenté/remplaçant) + avantages sociaux, } \\
\text { productivité réduite due aux dommages } \\
\text { matériels, pénalité de retard de livraison. }\end{array}$ & $\sqrt{ }$ & V & v \\
\hline Coûts salariaux & $\begin{array}{l}\text { Primes de surtemps et hausse des salaires } \\
\text { (dues au risque) + avantages sociaux. }\end{array}$ & $\sqrt{ }$ & V & $v$ \\
\hline $\begin{array}{l}\text { Frais } \\
\text { administratifs } \\
\text { indirects }\end{array}$ & $\begin{array}{l}\text { Frais administratifs de l'assureur privé et } \\
\text { public, frais de recrutement/dotation, de } \\
\text { formation, d'investigation de l'accident et de } \\
\text { suivi de dossier + avantages sociaux. }\end{array}$ & & v & $\sqrt{ }$ \\
\hline Frais judiciaires & $\begin{array}{l}\text { Expertises et contestations médicales, } \\
\text { défenses de dossier et poursuites. }\end{array}$ & & & $\sqrt{ }$ \\
\hline Réputation & $\begin{array}{l}\text { Pertes de contrats, difficulté à recruter, effets } \\
\text { sur réputation (augmentation de frais } \\
\text { marketing, commerciaux). }\end{array}$ & & v & \\
\hline $\begin{array}{l}\text { Autres frais (ou } \\
\text { crédits) }\end{array}$ & $\begin{array}{l}\text { Frais non assurés, réparation/remplacement } \\
\text { de fourniture/machine, nettoyage, chauffage/ } \\
\text { électricité, location, correctifs suite à } \\
\text { l'accident (R\&D), subvention R\&D, prix, } \\
\text { récompenses, crédit d'impôts, brevet } \\
\text { d'invention. }\end{array}$ & & $\sqrt{ }$ & $\sqrt{ }$ \\
\hline
\end{tabular}

71 Certes nos résultats sont basés sur les analyses de cinq cas faites par 22 étudiants futurs gestionnaires en ressources humaines, des analyses faites dans un environnement contrôlé, un séminaire de deuxième cycle universitaire de gestion des effets d'interaction entre les problèmes de santé et de sécurité au travail et de gestion des ressources humaines. De plus, ces résultats comportent des limites importantes, ils ne portent que sur des entreprises de la région métropolitaine. L'échantillon n'est aucunement représentatif des secteurs économiques et des types de lésions rencontrées dans les entreprises du Québec.

72 Étant donné les résultats de ce projet, nous proposons de peaufiner l'outil d'apprentissage en élargissant le nombre de cas, pour diversifier les profils 
d'entreprises et les événements ayant conduit à des analyses de coûts. Cet outil combine la Grille simplifiée d'analyse des coûts d'une lésion professionnelle et les trois scénarios de situation : réelle, aggravée et menaçante. Nous suggérons que cet outil soit testé dans deux contextes d'apprentissage : des ateliers de formation continue adressés aux gestionnaires en exercice et des séminaires de formation adressés aux futurs gestionnaires (étudiants). Pour les deux contextes d'apprentissage (atelier et séminaire), nous recommandons de dédier une partie du temps à l'étude de cas en petite équipe, et une partie du temps à l'analyse comparée des coûts et des décisions d'investir ou non lors de discussions en grand groupe. L'idée est de simuler le travail décisionnel des comités paritaires de SST et des directions au sein des entreprises.

\section{BIBLIOGRAPHIE}

Baril-Gingras, G., Bellemare, M., Poulin, P., Ross, J. (2010). Conditions et processus de changement lors d'interventions externes en SST. Élaboration d'outils pour les praticiens. Rapport R-647. Institut de recherche Robert-Sauvé en santé et en sécurité du travail.

Bérubé, M. (2004). Fiche technique \#46. Organisation de la prévention : la démarche préventive : niveaux d'intervention. Association paritaire pour la santé et la sécurité du travail, secteur des affaires municipales. www.apsam.com/sites/default/files/docs/publications/ft46.pdf.

Boulenger, S., Vaillancourt, F. (2012). Coûts des régimes de SST : méthodologie et résultats de la comparaison entre la CSST du Québec et la CSPAAT de l'Ontario. CIRANO, 2012RP-03.

Bräunig, D., Kohstall, T. (2011). Rendement de la prévention : Calcul du ratio coût-bénéfice de l'investissement dans la sécurité et la santé en entreprise. Résumé des résultats. Responsables scientifiques Association internationale de la sécurité sociale. Genève. http://acorfrance.ocnc.fr/IMG/pdf/Rendement_de_la_Prevention.pdf

Brody, B., Létourneau, Y., Poirier, A. (1990). Le coût des accidents du travail : État des connaissances. Relations industrielles, 45, 1, 94-117. www.erudit.org/revue/ri/1990/v45/ n1/050562ar.pdf

Brouselle, A., Champagne, F., Contandriopoulos, A.P., Hartz, Z. (2009). L'évaluation : concept et méthodes. Les Presses de l'Université de Montréal.

Brun, J.-P., Lamarche, C. (2006). Évaluation des coûts du stress au travail. Rapport de recherche. Chaire en gestion de la santé et de la sécurité du travail dans les organisations. Université Laval. http:// cgsst.com/wp-content/uploads/2016/07/\%C3\%89valuation-des-co\%C3\%BBts-du-stress-autravail.pdf

Cadieux, J. et Pérusse, M. (2018). Culture de SST - Qu'en est-il au Québec ?, Travail et Santé. https://travailetsante.net/articles/culture-de-sst-qu-en-est-il-au-quebec/

Cadieux, J., Roy, M., Desmarais, L. (2004). Changement de perspective dans la mesure de performance en SST. Chaire d'étude en organisation du travail. Université de Sherbrooke. www.researchgate.net/ profile/Lise_Desmarais/publication/ 
228423633_Changement_de_perspective_dans_la_mesure_de_performance_en_SST/links/ 00b49528e2892635cf000000.pdf

Commission de la construction du Québec (2017). Salaire. Taux et cotisations pour la période du 26 février 2017 au 25 mars 2017. 610 - Manœuvre canalisation souterraine. www.ccq.org/fr-CA/ M06_Salaire ?profil =GrandPublic

Champoux, D., Brun, J.-P. (2010). Dispositions, capacités et pratiques de SST dans les petites entreprises : opinions de patrons, d'employés et d'intervenants en SST au Québec. PISTES, 12, 2. https://pistes.revues.org/2525

Centre patronal de santé et sécurité du travail du Québec. (2000). Histoire de la santé-sécurité du travail au Québec : revenir sur le passé pour comprendre le présent et planifier le futur! Convergence, 16, 1, 4-5. www.centrepatronalsst.qc.ca/documents/pdf/conv_janv_00.pdf

Centre patronal de santé et sécurité du travail du Québec. (2005). Les outils du comité de santésécurité. Guide pratique. www.centrepatronalsst.qc.ca/documents/pdf/guide_outils_comite.pdf CNESST (2016). Rapport annuel de gestion 2015. www.centredoc.csst.qc.ca/pdf/ Rapports_annuels_CSST_et_annexes_statistiques/Rapport_annuel_2015.pdf

CSST (2007). Pour comprendre le régime québécois de santé et de sécurité du travail. www.cnesst.gouv.qc.ca/publications/200/Documents/DC_200_256_14.pdf

CSST (2011). La modernisation du régime de santé et sécurité du travail. www.cnesst.gouv.qc.ca/ publications/Documents/ Modernisation_du_r \%C3\%A9gime_de_sant \%C3 \%A9_et_s \%C3\%A9curit \%C3 \%A9_du_travail.pdf Darbon, S., Letourmy, A. (1983). La micro-économie des soins médicaux doit-elle nécessairement être d'inspiration néo-classique ? Sciences sociales et santé, 1, 2, 31-77. www.persee.fr/docAsPDF/ sosan_0294-0337_1983_num_1_2_940.pdf

Daures, J.-P., Guy Delande, (2000). L'évaluation médicoéconomique : objectifs, méthodologie, limites. Gastroentérologie Clinique et Biologique, 24, 11, 1029-1031. www.em-consulte.com/ showarticlefile/98358/index.pdf

Directeur de la santé publique de Montréal (2016). Les travailleurs invisibles. Les risques pour la santé des travailleurs des agences de location de personnel. Direction régionale de santé publique CIUSSS du Centre-Sud-de-l'île-de-Montréal. https://publications.santemontreal.qc.ca/uploads/ tx_asssmpublications/978-2-550-77210-1.pdf

Drummond, M., Sculpher, M., Torrance, G. (2005). Méthode d'évaluation économique des programmes de santé (3e édition). New York, Oxford University Press.

Eakin, J.M. (1992). Leaving it up to the workers : sociological perspective on the management of health and safety in small workplaces. International Journal of Health Services, 22, 4, 689-704.

Ferron, M.A (2013). Gérez la santé sécurité - Obtenez le meilleur, prévenez le pire. Édition Question Authentique, Québec.

Frick, K., Kempa, V. (2011). Les systèmes de gestion de la sécurité et de la santé au travail. Quand sont-ils bons pour votre santé ? Rapport 119, Institut syndical européen. www.etui.org/content/download/ 7282/66379/file/rapport-119-FR.pdf

Gaudemet, J.-P., Walliser B. (1983). Méthodologie de l'évaluation des effets macroéconomiques de projets microéconomiques ou d'interventions publiques spécifiques. Économie \& Prévision, 60, 3-31. www.persee.fr/doc/ecop_0249-4744_1983_num_60_4_3271 
Gilks, J., Logan. R. (2010). Les accidents du travail et les maladies professionnelles au Canada, 1996 - 2008. Taux des accidents de travail et leur coût de l'économie. Recherche et analyse. Division de la santé et sécurité au travail, Programme du travail, Ressources humaines et Développement des compétences Canada. www.travail.gc.ca/fra/sante_securite/pubs_ss/pdf/atmc.pdf

Gouvernement du Québec ; Affaires municipales et Occupations du territoire. (2010). Organisation territoriale. Régions administratives. Québec : l'auteur. www.mamot.gouv.qc.ca/organisationmunicipale/organisation-territoriale/regions-administratives/

Gosselin, M. (2004). Analyse des avantages et des coûts de la santé et de la sécurité au travail en entreprise Développement de l'outil d'analyse. Rapport R-375. IRSST, www.irsst.qc.ca/media/ documents/pubirsst/R-375.pdf

Gravel, S., Bernstein, S., Villanueva, F., Hanley, J., Crespo, D. (2017). Le recours à l'embauche des travailleurs étrangers temporaires dans les secteurs saisonniers au Québec : le point de vue des employeurs. Canadian Ethnic Studies, 49, 2, 75-98.

Gravel, S., Lortie, M., Bilodeau, H., Dubé, J. (2013). L'interaction entre les problèmes de gestion des ressources humaines et de santé et de sécurité au travail. Montréal : Presses de l'Université du Québec, collection santé et société, 222 pages. www.puq.ca/catalogue/livres/interaction-entre-lesproblemes-gestion-des-17634.html

Grimaldi, J.V., Simonds R.H. (1989). Safety management (5th edition). R. D. Irwin. : Éditions Homewood.

Heinrich, H.W. (1941). Industrial accident prevention a scientific approach (2nd edition). New York, New York McGraw-Hill.

Hoch, J.S., Dewa C. (2005). An introduction to economic evaluation : what's in a name? Can J Psychiatry, 50, 3, 159-166.

Institut de la statistique du Québec (2001). Portrait social du Québec. Données et analyses. Collection les conditions de vie. Québec, l'auteur. www.stat.gouv.qc.ca/statistiques/conditions-vie-societe/ portrait-social2001.pdf

Institute for Work \& Health (2010). Health \& Safety Smart Planner : Economic Evaluation Software. www.iwh.on.ca/smart-planner

Koninckx, G., Teneau G. (2010). Résilience organisationnelle : rebondir face aux turbulences. Bruxelles, De Boeck, 295 p.

Lanoie, P., Rivard, C., Fortin, M., Bernard, S., Bouchard, I., Dubuc, M.F., Seudjio, J., Tremblay, L., Zenadocchio, N. (2019). Audit de performance, Prévention en santé et sécurité du Travail ; Commission des normes, de l'équité, de la santé et de la sécurité au travail. Chapitre 3, Rapport du commissaire au développement durable, Rapport du Vérificateur général du Québec à l'Assemblée nationale pour l'année 2019-2020.

Laroche, E., Dionne-Proulx, J., Legault, M.-J. (2013). Gestion de la santé et de la sécurité au travail. Montréal, Chenelière Éducation.

Laufer, A. (1987). Le coût des accidents du travail. État des connaissances. Relations industrielles, $45,1,94-116$

Lebeau, M. (2015). Coûts des lésions professionnelles. DS-013, Institut de recherche Robert-Sauvé en santé et en sécurité du travail. www.irsst.qc.ca/media/documents/PubIRSST/DS-013.pdf

Lebeau, M., Duguay, P. (2011). Les coûts des lésions professionnelles. Revue de littérature. Rapport R-676, IRSST. www.irsst.qc.ca/media/documents/PubIRSST/r-676.pdf 
Lebeau, M. Duguay, P., Boucher, A. (2011). Estimation des coûts des lésions professionnelles. Une étude de faisabilité dans le secteur minier. Rapport R-717, IRSST. www.irsst.qc.ca/media/documents/ PubIRSST/R-717.pdf

Lebeau, M., Duguay, P., Boucher, A. (2014). Les coûts des lésions professionnelles au Québec, 2005-2007. Rapport R-769, IRSST. http://irsst.qc.ca/media/documents/PubliRSST/R-769.pdf

Lee G. (2017. A Systematic Review of Occupationnal Health and Safety Business Cases. Workplace Health \& Safety, 66, 2, 95-104.

Lejeune, C. (2008). L'évaluation économique, un outil d'aide à la décision médicale. Médecine.

Millette, J. (2013). Le coût des lésions professionnelles Quels coûts considérer ? Pour qui ? Bâtir les connaissances. Prévention au travail, 26, 3. www.preventionautravail.com/images/archives/ 26-03.pdf

Ordre des psychologues du Québec (2016). Combien coûte une psychothérapie. www.ordrepsy.qc.ca/ web/ordre-des-psychologues-du-quebec/combien-ca-coute-

Organisation mondiale de la Santé (2003). Économie de la santé. Principe d'évaluation économique pour les responsables des programmes de contrôle des maladies tropicales. Organisation mondiale de la Santé VIH/SIDA, tuberculose et paludisme. Faire reculer le paludisme. Édition Provisoire. www.who.int/ malaria/publications/atoz/economics-fr_lg.pdf ?ua $=1$

Prevor (2016). La solution Diphotérine - solution de lavage d'urgence des lésions chimiques. Prevor. Prévoir et Sauver. Laboratoire de Toxicologie et Maîtrise du Risque Chimique. www.prevor.com/ fr/la-solution-diphoterine-traitement-des-lesions-chimiques

Racila, V. (2016). Résultats de l'Enquête sur la rémunération globale au Québec. Collecte 2015. Institut de la statistique du Québec. www.stat.gouv.qc.ca/statistiques/travail-remuneration/resultatserg-2015.pdf

Roy, M., Cadieux, J., Desmarais, L. (2005). Améliorer la performance en SST : les résultats vs les prédicteurs. PISTES, 7, 2. http://pistes.revues.org/3214

Sultan-Taïeb, H. (2011). Analyse coût-avantage des interventions de prévention du stress au travail. Stress au travail et santé, situation chez les indépendants. Collection Expertise collective. France : Éditions Inserm. www.ipubli.inserm.fr/bitstream/handle/10608/217/Chapitre_17.html.

Sylvestre, C. (2017). Analyse des coûts microéconomiques et santé et sécurité au travail ; application d'une grille d'analyse à cinq études de cas d'entreprises. Mémoire présenté comme exigence partielle de la maîtrise ès de la gestion, Université du Québec à Montréal, 193 pages.

Tangri, R. (2003). Stress Costs Stress-Cures. Halifax : Chrysalis Performance Strategies Inc. www.secretsellingtips.com/Vault/StressCostsStressCures.pdf

Tompa, E., Dolinschi R., de Oliveira, C. (2006). Practice and potential of economic evaluation of workplace based for occupational health and safety. J Occup Rehabil, 16, 375-400.

Tompa, E., de Oliveira C., Dolinschi R., Irvin E. (2008). A Systematic Review of Disability Management Interventions with Economic Evaluations. J Occup Rehabil, 18, 16-26.

Tompa, E., Verbeek, J., Van Tulder M., De Boer, A. (2010). Developing guidelines for good practice in the economic evaluation of occupational safety and health interventions. Scand J Work Environ Health, 36, 4, 313-318.

Uegaki, K., De Bruijne, M.C., Van Der Beek, A. J., Van Mechelen, W., Van Tulder, M., W. (2010). Economic evaluations of occupational health interventions form a company's perspective : a 
systematic review of methods to estimate the cost of health related productivity loss. J Occup Rehabil.

Vinet, E. (2015). Les pratiques gagnantes en gestion et SST. État de la question. Institut de recherche. IRSST. http://medias.irsst.qc.ca/videos/1505_et_cr_HD_modeleGestionSST_fr_pdf.pdf

\section{NOTES}

1. www.iwh.on.ca/tools-and-guides/health-safety-smart-planner-economic-evaluation-software.

2. www.iwh.on.ca/newsletters/at-work/66/iwh-tools-in-action.

3. Un événement violent ayant une présomption médicale de chocs post-traumatiques estimé en moyenne à 45000 \$ par événement (donnée basée sur 2165 lésions professionnelles à cause des actes violents perpétrés entre 2005 à 2007) (Duguay et Boucher; 2014).

4. Prevor, 2016.

\section{RÉSUMÉS}

Cet article présente les résultats d'un outil d'apprentissage à la gestion de la santé et de la sécurité du travail (SST) basé sur les coûts. Le projet s'est déroulé en deux temps : 1) élaborer, avec des gestionnaires en exercice, cinq études de cas centrés sur les coûts $(n=5)$ à partir d'un accident ou d'une maladie d'origine professionnelle; 2) tester les capacités des étudiants en gestion $(n=22)$ à identifier les coûts des événements et à anticiper les coûts de situations aggravées. Après avoir étudié trois cas, les étudiants peuvent facilement identifier et calculer les coûts directs et indirects associés aux salaires et aux pertes de production, mais sous-estiment les coûts de remplacement des employés et de gestion de la SST. Ils peuvent facilement anticiper les coûts de situations aggravées par des menaces syndicales. Cet outil d'apprentissage pourrait être transposé en un atelier de formation continue et en un contenu obligatoire de formation.

This article presents the results of a cost-based occupational health and safety (OHS) management learning tool. The project was conducted in two phases: 1) developing, with practicing managers, five cost-focused case studies $(n=5)$ of work-related accidents or illnesses ; 2) testing the abilities of management students $(n=22)$ to identify the costs of an event and anticipate the costs of an aggravated situation. After studying three cases, students could easily identify and calculate the direct and indirect costs associated with wages and lost production, but underestimated the costs of employee replacement and OHS management. Likewise, they could easily predict the costs of situations aggravated by union threats. This learning tool could be included in continuing education workshops and mandatory training. 
INDEX

Keywords : occupational health and safety, cost analysis, occupational injuries, learning tool, repair costs

Mots-clés : santé et sécurité au travail, analyse des coûts, lésions professionnelles, outil d'apprentissage, coûts de réparation

\section{AUTEURS}

SYLVIE GRAVEL

École des sciences de la gestion, Université du Québec à Montréal, Québec, Canada JESSICA DUBÉ

Institut de recherche Robert-Sauvé en santé et en sécurité du travail, Québec, Canada

\section{CAROLE SYLVESTRE}

École des sciences de la gestion, Université du Québec à Montréal, Québec, Canada 